\title{
我和汉学的不解之缘一一采访 The Indissoluble Bound with 西班牙格拉纳达大学佩德罗・Sinology: An Interview with 圣•赫内斯・阿吉拉教授 Professor Pedro San Ginés Aguilar from the University of Granada, Spain
}

\section{Pedro San Ginés Aguilar}

Profesor de la Universidad de Granada y doctor en Filología románica. Estuvo Licenciado en estudios chinos por la Universidad de París 8 (1976) y fue ex director del Instituto Confucio de la UGR, y también es Asesor del Instituto Confucio de la UGR. Desempeña el cargo de Director del Grupo de Investigación de estudios Asiáticos GIDEA - ex coordinador del Foro de Estudios asiáticos (FEIAP), además de ser Coordinador del Grado chino en el Grado de Lenguas Modernas y sus Literaturas. Es Presidente de la Asociación Andaluza Asia y Director de la colección interlingua de la editorial Comares de Granada y Director de la colección de Estudios Asiáticos de la misma editorial. Las obras publicadas incluyen Cruce de

\footnotetext{
" [作者简介] 解雅楠, 天津外国语大学在读硕士。研究方向: 东方文学。

[基金项目］本文为国家社会科学基金项目 “中国文学在西班牙的翻译与传播研究”（14CWW027）的 阶段性成果。
} 
我和汉学的不解之缘——对西班牙格拉纳达 The Indissoluble Bound with Sinology: An 154 大学佩德罗・圣・赫内斯・阿吉拉教授的采访 Interview with Professor Pedro San Ginés Aguilar...

miradas, relaciones e intercambios, Introducción a la traducción jurídica y jurada, La investigación sobre Asia pacífico en España, Planteamientos generales de la traducción, etc.

Entrevistado: Pedro San Ginés Aguilar

Entrevistadora: Xie Yanan

Fecha de la entrevista: el 3 de abril de 2016

Lugar de la entrevista: Universidad de Granada

Objetivo de la entrevista: el comienzo de enseñanza de chino en Granada

Xie Yanan: ¿Naciste en Francia?

Pedro San Ginés Aguilar: -Nací en Francia en 1949, es curioso el año de la Fundación de la República Popular China.

Xie Yanan: ¿Tus padres ambos son españoles? ¿Por qué fueron a Francia?

Pedro San Ginés Aguilar: -Mis padres eran españoles, comunistas y republicanos, exiliados en Francia desde 1939. También participaron en la defensa de Francia contra el nazismo en la Guerra Mundial.

Xie Yanan: ¿Por qué te surgió la idea de aprender chino?

Pedro San Ginés Aguilar: -Primero, estudié “Ingeniería Técnica en Calefacción y Aire Acondicionado", acabando en el año 1968. Tenía 19 años y empecé a trabajar como profesional hasta 1977 cuando ya emprendí una nueva vida en Granada. Sin embargo, en 1968 ocurrió un movimiento estudiantil muy profundo en "mayo" al que participé. Sería quizás largo de explicar, pero el movimiento comunista se puede decir que se dividió en dos tendencias: una prosoviética y otra maoísta. Aquellos jóvenes del" mayo del 68" éramos más de tendencia maoísta. Seguramente fue la consecuencia de la división entre China y la Unión soviética de los años 60 .

-Estaba trabajando cuando decidí volver a estudiar. Había nacido una nueva universidad llamada "Vincennes", hoy París 8. Era una Universidad que nacía del movimiento estudiantil del 68, y abrió sus puertas en 1969. Era un centro experimental. Ingresé en la Universidad en 1970 ó 1971 (tengo que averiguar). Era una universidad fantástica y los más importantes pensadores de la época en muchos campos pasaron por sus aulas (entre ellos Michel Foucault). Empecé con los estudios “Hispánicos” y 
al año siguiente hice una prueba con una asignatura optativa de lengua china que me atraía mucho (por el aspecto ideológico y cultural), sobre todo al saber que había estudios sinológicos en la universidad. Llevé adelante, pues, dos licenciaturas, al mismo tiempo que trabajaba. En 1975, acabé la licenciatura de hispánicas y en 1976 la de chino. En 1976 obtuve la tesina (hoy sería un examen de máster) de hispánicas y en 1977 la de chino.

Xie Yanan: ¿Quién era tu profesora en el estudio sinólogo en Francia?

Pedro San Ginés Aguilar: - Mi maestra fue la profesora Michelle Loi (se puede encontrar un resumen de su vida en Internet). Una mujer extraordinaria, era muy amiga del famosos filósofo marxista Louis Althusser quien habla de ella en uno de sus libros. Ella me dirigió mi tesina sobre "Teatro chino revolucionario". Fue de pensamiento maoísta de la época. Iba a dirigirme mi Tesis sobre "Teatro en China", pero ya le dije que me iba a Granada. Continuamos una relación excelente y la recordaré siempre como una magnífica profesora y maestra. Su forma de enseñar y su capacidad crítica ha influido mucho en mí. Falleció en 2002. Era especialista de Lu Xun e hizo muchas traducciones y artículos sobre este escritor y en defensa de la mujer.

Xie Yanan: ¿Por qué volviste a España? ¿En qué año?

Pedro San Ginés Aguilar: -Conocí a mi mujer en un viaje de verano por España, y en 1976 nos casamos en la región parisina. Allí vivimos un año para acabar mi tesina de chino en 1977. Decidimos venirnos a Granada, dejando mi trabajo y empezando una nueva vida sin saber lo que iba a pasar. En realidad, no volví a España, puesto que había nacido en Francia y toda mi juventud y todos mis estudios fueron franceses. Mi cultura principal, pues, fue francesa. Es cierto, la influencia española de mis padres fue muy importante. Mi hermano y hermanas viven en Francia y son franceses.

Xie Yanan: ¿Cómo llegaste a Granada y empezaste la enseñanza de chino? ¿En qué año?

Pedro San Ginés Aguilar: - Una vez instalados en Granada en junio de 1977, empecé a trabajar en escuelas privadas de idiomas, enseñando francés. En 1977, también estuve creando, en la escuela de Artes y Oficios de Granada un taller de marionetas que reanudaba con la tradición 
我和汉学的不解之缘——对西班牙格拉纳达 The Indissoluble Bound with Sinology: An 156

大学佩德罗・圣・赫内斯・阿吉拉教授的采访 Interview with Professor Pedro San Ginés Aguilar...

granadina de los años 30. Montamos la obra de García Lorca "Doña Rosita la Soltera o el lenguaje de las flores". Constaba de 28 marionetas de hilo. Estuvimos representando el espectáculo en Granada y en el sur de Francia. El grupo se llamó “Totolín”, una marioneta creada por Hermenegildo Lanz, amigo de García Lorca y Manuel de Falla, y el que creó las "marionetas de Lorca y la escenificación del retablo de Maese Pedro de falla". Su nieto Enrique Lanz aprendió conmigo las marionetas y hoy es quizás el mejor de los marionetistas de España. Montó él mismo el "retablo de Maese Pedro". Ver la página Web http://titeresetcetera.com/.

-Fui al Rectorado de la UGR a principios de 1978 para pedir enseñar chino. Me dijeron que si había 10 alumnos que se matriculaban en el Instituto de Idiomas estaba contratado, y así fue. Las enseñanzas del chino empezaron, pues, durante el curso 1978-1979.

Xie Yanan: ¿Cómo te surgió la idea de promover la traducción de pabellón en sueño rojo? ¿y cómo ha sido el proceso?

Pedro San Ginés Aguilar: -A raíz del inicio de la enseñanza del chino, tomé contactos con la Embajada China en Madrid. Se portaron magníficamente conmigo y el proyecto de enseñanza del chino. Unas de las personas de la Embajada y su marido se ocupaban de la cultura. Y quiero aquí recordar a Mme. Ma (De formación francesa. No recuerdo el nombre de su marido), una mujer que nos ayudó muchísimo. Solo la conozco por ese nombre. Realizamos exposiciones sobre China que llevamos de un pueblo a otro, proyectamos películas. Vinieron las primeras delegaciones de la república Popular China. ¡Fue una época increíble e ilusionante!

- Si uno recuerda la época, en España no había nada sobre China, y en Granada menos. Era alguien extraño. La Embajada empezaba su andadura en una España que pasaba del fascismo a la democracia, y China empezaba igualmente una nueva andadura con Deng Xiaoping. Ambos países iniciaban un nuevo camino. A pesar de las dificultades económicas de China en la época, me ayudaron de todas las maneras. Incluso, un día me dieron hasta bonos de gasolina. Fue una muestra de una gran generosidad cuando todavía China no era la potencia económica que es hoy. Las relaciones estaban establecidas y planteamos la posibilidad a través de la Embajada de unir Beida (Universidad de Pekín) con Granada. Estamos en 1979. El Rector era Antonio Gallego Morell y el Secretario General Fermín Camacho quien fue a hablar con dicha embajada.

-Era 1980, y el Instituto de Idiomas en el que daba clases de chino se iba a transformar en "Escuela Universitaria de traducción e 
Interpretación", y más adelante en Facultad (no recuerdo la fecha de su creación). Fue un proceso lento en el que el chino iba a ser segunda lengua extranjera, como lo es todavía hoy. En los años 80 , pues, daba clases en traductores y letras.

- Los contactos con China a través de la Embajada se iban concretando, y en una fiesta de la Embajada China en Madrid (creo que año nuevo, y no recuerdo la fecha), se decidió la "Traducción del Sueño en el pabellón Rojo", y las relaciones con la Editorial de lenguas extranjeras de Beijing.

- Los cambios iban a empezar en las Universidades españolas y la elección del Rector se iba a realizar por elección de todos sus miembros. El primer Rector que ganó la elecciones en 1985 fue José Vida Soria quien formó parte de la “Comisión Constitucional Mixta Congreso-Senado, encargada de dar la última versión de la Constitución española de 1978. Con la llegada en 1985 del nuevo Rector José Vida Soria, el Secretario General Juan Francisco García Casanova e, igualmente el Vicerrector de extensión universitaria de la época Juan José Ruiz-Rico, se inicia la profundización de los contactos y los estudios del chino. Se decide la creación de un convenio con Beida y se inicia con el intercambio de profesores.

-La primera profesora elegida sería Ángela Olalla. Me habían propuesto que fuera yo, pero no podía, puesto que, si me iba un año, se perdía la enseñanza de la lengua china. Tras el retorno de Ángela Olalla, nos fuimos una delegación de la UGR a Beijing, las tres personas citadas anteriormente (Rector, Secretario General y Vicerrector) y yo mismo. Firmamos el acuerdo para el intercambio de profesores.

-Los primeros profesores en llegar a Granada fueron el profesor Shen Shiyan y Duan Yuran (creo que así se llamaba). ¡Hicieron una labor inconmensurable! Recuerdo al Profesor Shen como un Gran Profesor y un amigo entrañable. Siempre estará en mi corazón.

-Cuando se me propuso la traducción del "Sueño en el Pabellón Rojo", se decidió que un profesor del departamento de hispánica de Beida iba a realizarlo. Como tardaba en venir el profesor y no se sabía nada, el Secretario General Juan Francisco García Casanova dijo sí a la petición de Zhang Zhengquan de la Universidad de Xi'an para que viniera para iniciar la traducción del libro. Al final, vino el profesor Zhao Zhenjiang quien iba a realizar un trabajo magnífico y muy duro. Se resolvió el problema de los dos profesores chinos, puesto que el profesor Zhang iba a dar clases en Traductores y Zhao se ocuparía totalmente de la Traducción. 


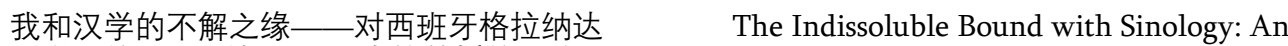
158 大学佩德罗・圣・赫内斯・阿吉拉教授的采访 Interview with Professor Pedro San Ginés Aguilar...

-Recuerdo nuestros primeros debates sobre los problemas de traducción de esta obra universal. Por ello, Zhao también quedará en mis recuerdos más queridos. Como yo estaba con mi Tesis Doctoral, decidimos que debía haber alguien que se ocupara, junto al profesor Zhao, de la revisión del español. Fue la profesora Ángela Olalla quien sugirió a José Antonio García Sánchez (conocido como el Murciano). Así fue y los dos colaboraron estrechamente, creándose una profunda amistad entre ambos.

-Cierto, es que, en un principio, trabajé en los dos primeros capítulos de la obra. Pero mi dedicación a la Tesis doctoral me impedía hacer más, así como mi dedicación en la construcción del futuro en las enseñanzas de la lengua y cultura chinas.

Xie Yanan: ¿Cómo encontraste a Alicia Relinque, Juanjo y José Javier para que ellos participaran en este equipo de estudios chinos en UGR?

Pedro San Ginés Aguilar: -Cuando el profesor Zhang Zhengquan dio las clases de chino en Traductores, me fui definitivamente a la Facultad de Filosofía y Letras a dar "lengua francesa".

-Con las nuevas reformas universitarias, y el apoyo del Decano Miguel Gómez Oliver, se decidió fomentar el chino. Tras leer mi Tesis Doctoral en 1989 sobre Teoría de la traducción, pedí mi traslado al departamento de Lingüística General y Teoría de la Literatura y pasar mis oposiciones a Profesor Titular en el área de Lingüística general en el que iba a crearse los estudios sinológicos dentro del área de Lingüística general. En estos años ochenta, también crea la "Asociación universitaria de Lengua y cultura chinas”. En 1991 estábamos con la nueva Decana Cándida Martínez cuando se aprobó que fuera a oposición en Lingüística General con el perfil "Pensamiento chino en la lengua y escritura chinas" (no recuerdo bien el perfil exacto). Gané las oposiciones y empezamos las primeras asignaturas de cultura china en el curso 1991-1992.

- La profesora Ángela Olalla me sugirió que dirigiera la petición de Tesis de Alicia Relinque Eleta sobre una traducción de la obra de teoría literaria de Liu Xie "El corazón de la literatura y el cincelado de dragones". Acepté, y en julio de 1994 se leyó la Tesis con "Sobresaliente Cum laude". Unos años más tarde, la Decana Cándida Martínez, dentro de las reformas que se estaban realizando, me propuso un opcional de lengua china de 12 créditos. Como tenía demasiadas asignaturas, puesto que daba "Civilización china" también en traductores, pedí un profesor de chino, y tras un concurso de méritos, la comisión al que pertenecía contrató a Alicia 
Relinque Eleta (unos 5 años en China). Más adelante Juan José Ciruela que trabajaba en la Embajada española en Beijing me contactó para realizar sus estudios de doctorado y realizar su Tesis doctoral que presentó en 1999 "Historia de la lingüística china" con sobresaliente Cum laude. En cuanto a Gabriel García-Noblejas, realizó su Tesis, si no recuerdo mal en Oviedo, teniendo a Alicia Relinque como codirectora. Estuve en su tribunal de Tesis con el mismo resultado.

-Se necesitaban profesores en la Facultad de Traducción y en Letras. $Y$ como hice hasta el momento, consideraba que los profesores de chino que vendrían deberían saber mucho más la lengua china que yo. $\mathrm{Y}$ así se hizo, menos yo, todos los profesores hablan muy bien chino.

-En la convocatoria de plazas, se presentaron Juanjo y Gabriel, y Juanjo cedió su sitio a Gabriel (unos 5 años en China), puesto que en ese momento no podía venirse a Granada. Para el curso siguiente pudimos convocar nueva plaza y Juanjo (16 años en China) se incorporó al área de lingüística, mientras Gabriel se fue al área de Traducción en la Facultad de Traducción e Interpretación. En cuanto a José Javier, también realizó su Tesis doctoral sobre "la nueva cultura en China de los años 30 del siglo XX" en el año 2002 con sobresaliente Cum laude. Había vivido unos 8 años en China, y a su regreso dio clases de lengua china en el "Centro de Lenguas Modernas" de la UGR. Si no recuerdo mal, en 2006 se convocó una plaza Ayudante doctor que ganó José Javier. En su caso, fue el primer profesor que habíamos formado en Granada, cuando siguió las asignaturas de chino que se ofrecieron en China. Luego se fue como profesor de intercambio a Beida, continuo en diversos lugares de China como lector de español, formándose, a su vez, en lengua y cultura chinas.

-En 2004, dejé un grupo de investigación y creé el Grupo de Investigación de Estudios Asiáticos (GIDEA), al que pertenece Zhang, Juanjo, Gabriel y José Javier como profesores de Lengua y Cultura China.

Xie Yanan: ¿Aparte de esta nueva carrera de filología china, tenéis algún proyecto más?

Pedro San Ginés Aguilar: -En 2006, como miembro asesor de Casa Asia de Barcelona, pedí la financiación para la creación del "Foro Español de Investigación Asia Pacífico" (FEIAP), ver Internet. Reuní a mis compañeros y con ellos llevamos a cabo en Granada el "Primer Foro" que tuvo un gran éxito. El secretario designado fue Juan José Ciruela (Juanjo). Se creó una colección española de estudios asiáticos (CEIAP). Ver Internet. Los Foros iban a realizarse cada dos años en una ciudad española diferente. 
我和汉学的不解之缘——对西班牙格拉纳达 The Indissoluble Bound with Sinology: An 160

大学佩德罗・圣・赫内斯・阿吉拉教授的采访 Interview with Professor Pedro San Ginés Aguilar...

En 2008 fue en Valencia que acababa de poner en funcionamiento el primer “Instituto Confucio" en España con Vicente Andreu como primer director.

-Este mismo año, también pusimos en marcha el "Instituto Confucio" en Granada. En 2007 (tiene más información Alicia) se firmó un acuerdo entre la UGR y Beida para tal creación. En aquel entonces David Aguilar era Rector y Ángela Olalla y Alicia estuvieron en ese proyecto. Con el nuevo Rector Francisco González Lodeiro y la Vicerrectora de Relaciones Internacionales de la UGR Dorothy Kelly se puso en marcha el "Instituto". La Vicerrectora me pidió que llevara adelante el proyecto, cuando todavía no había nada. Fui, pues, el primer Director y tuve que construir desde cero lo que iba a ser el Instituto. Fan Ye fue el subdirector y trabajamos muy duro para levantar de la nada lo que hoy un buen Instituto que ha recibido un premio. Continúo siendo asesor a la Presidencia del Instituto Confucio. Fan Ye, fue invitado y participó en el $2^{\circ}$ Foro de Valencia.

-En 2010, se realizó el tercer Foro en Zaragoza y dejé, posteriormente, la coordinación del Foro, cediéndola a Juanjo. En febrero de este año se celebró el $4^{\circ}$ Foro en Granada, siendo el nuevo coordinador Juanjo y como Secretaria Sara Rovira de la Universidad Autónoma de Barcelona. Un nuevo camino se ha iniciado.

-En 2010, bajo la dirección de la Decana María Elena Martín Vivaldi se aprobó el "Grado de lenguas modernas y sus literaturas" que incluyen 4 lenguas: ruso, italiano, hebreo y chino. El "Grado de Lenguas Modernas y sus Literaturas, mención china" que coordino empezó su trayectoria en 2010. Mis sueños de estudios de chino que tenía en la cabeza en 1978, por fin se han realizado con esta licenciatura. Han sido un trabajo de 36 años para conseguir los objetivos deseados. He tenido que dejar mis intereses académicos propios para que eso pueda suceder. Si todo va bien, en el curso siguiente se iniciará el nuevo "Máster en estudios Asiáticos" que dirigirá Alicia.

- La asociación que creé en los años 80 ha cambiado de nombre y se llama "Asociación Andaluza Asia" (ASANAS) y continúo siendo su Presidente, y pretendemos lanzar su nueva página web muy pronto. Será un nuevo paso para la difusión del chino. También queremos con nuestro grupo de investigación (GIDEA) proponer proyectos de investigación que queremos realizar junto a Cevug (centro de la UGR) para la enseñanza on-line en lengua y cultura.

- Si queréis podríamos trabajar junto en algún proyecto de investigación entre nuestras dos universidades. 


\section{Conclusiones del entrevistado}

Tus preguntas me han traído muchos recuerdos en la memoria y mucha emoción, y también la tristeza de no haber podido estudiar en China por mi dedicación exclusiva a la construcción de los estudios sinológicos. Ya tenemos nuevas generaciones de sinólogos y creo que el esfuerzo ha valido la pena.

Quiero aquí recordar de nuevo a las dos personas que más han contribuido a la realización de este sueño: a Mme. Ma de la Embajada China de aquella época difícil, así como al profesor Shen Shiyan y al profesor Zhao Zhenjiang. Creo que los profesores de chino que llegaron al UGR son todos excelentes. Cada uno tiene su trayectoria y están aportando mucho a los estudios sinológicos españoles en traducciones, libros y artículos de muy buena calidad. Alicia en "Literatura clásica china"; Juanjo en "Lingüística china"; Gabriel en "Traducción del chino"; José Javier en "Literatura contemporánea china". Me siento orgulloso de todos ellos.

\section{Conclusión de la entrevista}

La experiencia universitaria en Francia durante la juventud le trajo a Profesor Pedro San Ginés Aguilar la oportunidad de conocer chino y su cultura, que constituye una cosa de vida, significativa e importante.

Al principio había aprendido chino en la Universidad de Paris 8, quien, más tarde, se convirtió a profesor impartiendo enseñanza a los alumnos en la universidad de Granada. Nunca ha perdido ninguna coyuntura sino aprovechó todo lo que pudiera a fomentar y promover el estudio sinológico en España ni a escatimar esfuerzos para llevar a cabo la formación de la siguiente generación. Profesor Pedro San Ginés, quien ha dedicado casi toda la vida al desarrollo de sinología española y de la relación académica sino-española, no sino es un gran maestro a quien deberíamos admirar y estimar para siempre. 